\title{
Symposium review: Single-step genomic evaluations in dairy cattle*
}

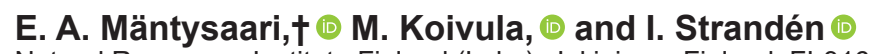

Natural Resources Institute Finland (Luke), Jokioinen, Finland, FI-31600

\begin{abstract}
During the last decade, genomic selection has revolutionized dairy cattle breeding. For example, Nordic dairy cows (Denmark, Finland, and Sweden) born in 2018 were $>90 \%$ sired by young genomically tested bulls. Thus, the average age of sires for Red Dairy Cattle cows born in 2018 was only 3.1 yr, whereas in 2011 it was 5.7 yr. Earlier the key driver of genetic progress was the selection of progeny-tested sires, but now it is the genomic preselection of young sires. This leads to a biased estimation of genetic progress by the traditional genetic evaluations. When these are used as input for multi-step genomic evaluations also they became distorted. The only long-term solution to maintain unbiasedness is to include the genomic information in evaluations. Although means for singlestep evaluation models were introduced in 2010, they have not yet been implemented in large-scale national dairy evaluations. At first, single-step evaluations were hindered by computational cost. This has been largely solved, either by sparse presentations of the inverses of the genomic relationship $(\mathbf{G})$ and pedigree relationship $\left(\mathbf{A}_{22}\right)$ matrices of genotyped animals needed in the single-step evaluation models based on $\mathbf{G}$ (ssGBLUP), or by using the single-step marker models. Approaches for $\mathbf{G}^{-1}$ are the APY-G, where the relationships among "young" animals are completely determined by their relationship to the "core" animals, and single-step evaluations where the $\mathbf{G}^{-1}$ is replaced by a computational formula based on the structure of $\mathbf{G}$ (ssGTBLUP). The single-step marker models include the marker effects either directly, as effects in the statistical model, or indirectly, to generate genomic relationships among genotyped animals. Concurrently with development of the algorithm, computing resources have evolved in both availability of computer memory and speed. The problems actively studied now are the same for both

Received October 15, 2019.

Accepted January 21, 2020.

*Presented as part of the Joint ADSA/Interbull Session: Breeding and Genetics: Ten Years of Genomic Selection at the ADSA Annual Meeting, Cincinnati, Ohio, June 2019.

†Corresponding author: esa.mantysaari@luke.fi
\end{abstract}

of the single-step approaches (GBLUP and marker models). Convergence in iterative solving seems to get worse with an increasing number of genotypes. These problems are more pronounced with low-heritability traits and in multi-trait models with high genetic correlations among traits. Problems are also related to the unbalancedness of pedigrees and diverse genetic groups. In many cases, the problem can be solved by properly accounting for contributions of the genotyped animals to genetic groups. The standard solving approach is preconditioned conjugate gradient iteration, in which the convergence has been improved by better preconditioning matrices. Another difficulty to be considered is inflation in genomic evaluations of candidate animals; genomic models seem to overvalue the genomic information. The problem is usually smaller in single-step evaluations than in multi-step evaluations but is more difficult to mitigate by ad hoc adjustments.

Key words: dairy cattle, genomic BLUP (GBLUP), evaluation bias, marker effect models

\section{INTRODUCTION}

In the last decade, genomic selection has become the main source of genetic progress in dairy cattle breeding. Because the selection in cattle can be much stronger in males than in females, the key driver in current breeding schemes is preselection of AI bulls. At the same time, the use of old progeny-tested bulls is becoming rare. In Denmark, Finland, and Sweden, dairy cows born in 2018 were $>90 \%$ sired by young genomic-tested bulls. Thus, the average age of sires for Red Dairy Cattle cows born in 2018 was only 3.1 years, whereas in 2011 it was still 5.7 years.

Most of the genomic enhanced breeding values (GEBV) in use are based on a multi-step approach, in which a reliably evaluated reference population is first used to estimate the values of SNP marker effects, and these are then used to calculate genomic predictions for the young animals. These populations can be called reference and candidate animals. The phenotypes for the reference animals are derived from national genetic evaluations or multiple trait across country evaluations (MACE) from Interbull. Thus, the reliability 
of genomic evaluations depends on the accuracy and unbiasedness of national evaluations. Generally, it is accepted that animal model evaluations are unbiased if the selection in the population is based on a linear function of observed data, and that this selection is realized inside fixed effect classes (see $\mathbf{L}^{\prime} \mathbf{X}=\mathbf{0}$ in Henderson, 1984). This is clearly violated when the ordinary animal model predictions (AM BLUP) are based only on phenotypes, pedigree, and classification of observations into fixed effect classes, but the most important selection decisions have been based on genomic information.

The genomic preselection bias problem can be solved, at least in theory, by incorporating the genomic information into ordinary animal models used in national evaluations (Mäntysaari et al., 2018). The method for this is called single-step genomic evaluation (ssGBLUP) and was published 10 years ago (Christensen and Lund, 2009, 2010; Aguilar et al., 2010). For the theory and advantages of the single-step evaluations, see the review in Legarra et al. (2014). The ssGBLUP have not been widely implemented in large-scale national dairy cattle evaluations due to fear of a too-large computational cost. Therefore, only a few studies have addressed the differences between animal model EBV and single-step GEBV. In the Nordic evaluation development, we have experimented with single-step evaluations since 2011 (Mäntysaari et al., 2011), although in the first attempts the phenotypes were deregressed EBV. The full single-step test-day evaluation results (Koivula et al., 2012a,b, 2015) did not show different genetic trends in EBV and GEBV among bulls with daughters. However, in more recent studies (Mäntysaa- ri et al., 2018; Masuda et al., 2018) the differences in trends become visible as the genomically selected bulls are increasingly having daughters. Figure 1 illustrates the trend estimates in 305-d protein production among Holstein bulls in Finland, Denmark, and Sweden. The trends are from a research data evaluation with 305-d milk, protein, and fat records from 3 lactations for 8.3 million Nordic cows. The GEBV were computed using ssGBLUP with 178,000 genotyped cows and bulls. The 2 regression lines were fitted on bulls born from 2010 to 2014. With the animal model EBV, the trend line, with slope of $1.8 \mathrm{~kg} / \mathrm{yr}$, seems to follow along the full time window, but the GEBV show a clear increase in trend (slope $4.2 \mathrm{~kg} / \mathrm{yr}$ ) after genomic preselection for young bulls became available in 2009. It has to be noted that the differences in the trend estimates are model and trait dependent. For example, a test-day model is likely to be better in estimating genetic and environmental trends than the lactation yield model, because in a testday model animals will have repeated observations in consecutive fixed effect classes.

The problems arising from genomic selection for animal model evaluations are well recognized, but most national dairy cattle evaluations are still based on a multi-step approach. Countries that participate in international sire evaluations and submit their genetic evaluation data to Interbull are obliged to report their evaluation methodology. In June 2019, only 1 country, the Czech Republic, reported use of full ssGBLUP to Interbull Genoforms (Interbull, 2019). Two others, the Walloon Region of Belgium (Colinet et al., 2013) and New Zealand (Winkelman et al., 2015), reported us-

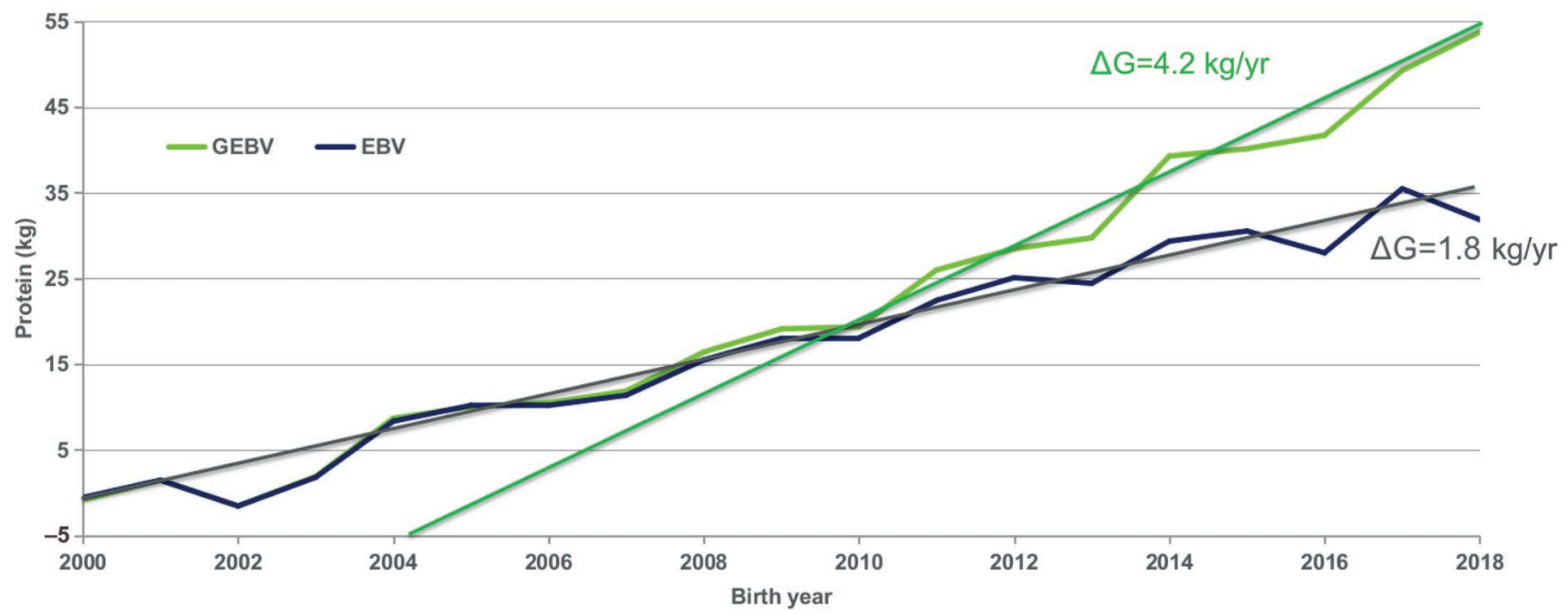

Figure 1. Average protein evaluations for Nordic (Finland, Denmark, and Sweden) Holstein bulls by birth year. EBV were estimated using an animal model for 305-d lactation records, and genomic enhanced breeding values (GEBV) with single-step genomic evaluations based on T matrix (Mäntysaari et al., 2017) from 178,000 genotyped animals (Koivula et al., unpublished data). 
ing so-called pseudo single-step, where the phenotypes in ssGBLUP were deregressed EBV from the national animal model.

The reasons for slow implementation of single-step evaluations are two-fold. The first challenge was computational. During the first years after the introduction of ssGBLUP, the method was considered impossible to compute in large populations with many recorded animals. In particular, the number of genotyped animals was thought to impose a definite limit for implementation. In the review paper by Legarra et al. (2014), the authors commented that methods to handle $>100,000$ genotyped animals exist but had not yet been tested. As better computational methods and models and ready-to-use software have become available, singlestep evaluations have started to appear for new traits with limited numbers of phenotypes or when no conventional national evaluations for the trait existed (Malchiodi et al., 2018; Gonzalez-Peña et al., 2019) and for other species than dairy cattle (Lourenco et al., 2018). The second reason for slow implementation has been the estimation bias. Most of the national dairy cattle genomic evaluations are certified for unbiasedness by the Interbull GEBV validation test (Mäntysaari et al., 2010). It has become apparent that single-step genomic evaluations can have problems passing validation tests and that special adjustments and model choices are needed (Tsuruta et al., 2019).

The main objectives of this review are (1) to give an overview of the current methods in single-step genomic evaluations and corresponding computational approaches for solving the model effects and (2) to discuss the problems noted in genomic predictions by single-step evaluations.

\section{SINGLE-STEP GENOMIC MODELS}

\section{ssGBLUP}

The first proposed single-step evaluations were based on the unified relationship matrix, which included genomic relationships for genotyped animals and modified pedigree relationships for other animals (Christensen and Lund, 2009, 2010; Aguilar et al., 2010). This was generally called ssGBLUP, reminding users that the matrix of genomic relationships $(\mathbf{G})$ was included in the BLUP framework. Although the unified relationship matrix $\mathbf{H}$ was complex and completely dense, it turned out that the inverse had a relatively simple form:

$$
\mathbf{H}^{-1}=\mathbf{A}^{-1}+\left[\begin{array}{cc}
0 & 0 \\
0 & \mathbf{G}^{-1}-\mathbf{A}_{22}^{-1}
\end{array}\right],
$$

where $\mathbf{A}$ is the pedigree-based additive relationship matrix and $\mathbf{A}_{22}$ is part of the $\mathbf{A}$ matrix for the genotyped animals. The solutions for GEBV were attained by replacing the $\mathbf{A}^{-1}$ matrix in the mixed-model equations (MME) by the $\mathbf{H}^{-1}$ matrix (see Legarra et al., 2014). No strict definition of the form of the genomic relationship matrix $\mathbf{G}$ was needed, although method I defined in VanRaden (2008) was used in the derivations. If the number of animals genotyped is $n_{g}$, the matrices $\mathbf{G}$ and $\mathbf{A}_{22}$, as well as their inverses, are $n_{g} \times$ $n_{g}$ dense matrices.

The $\mathbf{H}^{-1}$ matrix included the $\mathbf{G}^{-1}$ matrix. Because the $\mathbf{G}$ matrix derived using VanRaden method I is often singular, it was proposed to add a fraction of $\mathbf{A}_{22}$ to the $\mathbf{G}$ matrix to facilitate the inverse; that is, to use $\mathbf{G}_{w}=$ $(1-w) \mathbf{G}+w \mathbf{A}_{22}$, where $w$ would be a small arbitrary value (0.02 to 0.05 in Aguilar et al., 2010), or desired relative weight of the polygenic effect (Christensen and Lund, 2010). The $w$ value is commonly called the proportion of residual polygenic effect (RPG), and it can be interpreted to be a fraction of variation in the additive breeding values that cannot be described by the available markers. This can also be justified from the perspective of genetic modeling, because the finite number of markers might not accurately model the genomic relationships (Goddard et al., 2011).

\section{Single-Step Marker Effect Models}

Before the era of dense SNP marker arrays, genomic evaluations were based on marker effect models (MEM), but after the introduction of dense SNP data it became computationally more feasible to model the genomic breeding values than to fit the marker effects (Fernando et al., 2014). This was because the number of SNP was much greater than the number of genotyped animals. When single-step models were first introduced in 2010, the size of the matrices $\mathbf{G}$ and $\mathbf{A}_{22}$ was still manageable. However, it was apparent that when the number of genotyped animals will increase, the single-step models would eventually reach a limit where computing inverses of these matrices would not be possible.

\section{From Single-Step Marker Effect Model to Hybrid Model}

Fernando et al. (2013) suggested imputation of expected SNP genotypes to all non-genotyped animals and, after that, to treat all animals in the computations as genotyped. The proposed imputation was a simple linear prediction as in Gengler et al. (2007), which is as 
easy as fitting a simple animal model BLUP, SNP by SNP, for all the animals in the pedigree with the known genotypes as data. However, to take account of the uncertainty in the imputed genotypes, Fernando et al. (2013, 2014) included an extra model effect, called "imputation error," for the non-genotyped animals. In its simplicity, the resulting single-step marker effect model (ssMEM) was attractive and easy to understand, and it offered an easy way to model the marker effects using Bayesian regression (Fernando et al., 2014).

The drawback of the original ssMEM (Fernando et al., 2014) was the need for large intermediate data files. The computations were simple, but they required work files with as many rows as there were animals with observations, and as many columns with floating point numbers as there were SNP in the model. Taskinen et al. (2017) suggested storing only the true genotypes and imputation of the rest "on the fly" within each round of preconditioned conjugate gradient (PCG) iteration. Fernando et al. (2016) recognized the problem and proposed to build the SNP equations explicitly before iteration without actually storing the imputed SNP, and thus to avoid reading large data files during Monte Carlo sampling in Bayesian regression. They called this approach a single-step super hybrid model (ssHM). The same MME had been derived by Legarra and Ducrocq (2012), but they did not notice its computational efficiency.

The MME for the ssHM is

$$
\begin{aligned}
& {\left[\begin{array}{ccc}
\mathbf{X}^{\prime} \mathbf{X} & \mathbf{X}_{1}{ }^{\prime} \mathbf{W}_{1} & \mathbf{X}_{2}^{\prime} \mathbf{W}_{2} \mathbf{Z} \\
\mathbf{W}_{1}^{\prime} \mathbf{X}_{1} & \mathbf{W}_{1}^{\prime} \mathbf{W}_{1}+\lambda \mathbf{A}^{11} & \lambda \mathbf{A}^{12} \mathbf{Z} \\
\mathbf{Z}^{\prime} \mathbf{W}_{2}^{\prime} \mathbf{X}_{1} & \lambda \mathbf{Z}^{\prime} \mathbf{A}^{21} & \mathbf{Z}^{\prime} \mathbf{W}_{2} \mathbf{W}_{2} \mathbf{Z}+\lambda \mathbf{Z}^{\prime}\left(\mathbf{A}_{22}^{-1}-\mathbf{A}^{22}\right) \mathbf{Z}+\lambda m \mathbf{I}
\end{array}\right]} \\
& {\left[\begin{array}{c}
\hat{\mathbf{b}} \\
\hat{\mathbf{u}}_{1} \\
\hat{\mathbf{g}}
\end{array}\right]=\left[\begin{array}{c}
\mathbf{X}^{\prime} \mathbf{y} \\
\mathbf{W}_{1}^{\prime} \mathbf{y} \\
\mathbf{Z}^{\prime} \mathbf{W}_{2}^{\prime} \mathbf{y}
\end{array}\right],}
\end{aligned}
$$

presented in the form given in Appendix A in Legarra and Ducrocq (2012). Here, $\hat{\mathrm{g}}$ are the solutions for $m$ SNP effects; $\mathbf{X}, \mathbf{W}_{1}$, and $\mathbf{W}_{2}$ are the design matrices for fixed effects, breeding value effects for the nongenotyped animals, and direct genomic breeding values $(\mathbf{Z g})$ for the $n_{g}$ genotyped animals. The $\mathbf{Z}$ matrix is a centered matrix of marker effect covariables. Value $\lambda m$ is the ratio of residual variance over the SNP effect ( $g$ ) variance. Fernando et al. (2016) rewrote the diagonal block in Equation [2], pertaining to the marker solutions, as $\mathbf{Q}=\mathbf{Z} \mathbf{W}_{2}{ }^{\prime} \mathbf{W}_{2} \mathbf{Z}+\lambda \mathbf{Z}^{\prime} \mathbf{A}^{21}\left(\mathbf{A}^{11}\right)^{-1} \mathbf{A}^{12} \mathbf{Z}+\lambda m \mathbf{I}$, which they noted to be small enough $(m \times m$ matrix $)$ to be stored in memory during the iterative solving of MME.
The Fernando et al. (2016) ssHM model can be extended to accommodate additional random effects such as the residual polygenic effect. In a simple single-trait application, the number of random equations in Equation [2] is $m+\left(n-n_{g}\right)$, that is, the number of SNP plus the number of non-genotyped animals. If an RPG effect is included in the model, the number of random effect equations will be $m+\left(2 n-n_{q}\right)$. Such a model, however, includes 2 highly collinear breeding value effects for the non-genotyped animals, which is likely to cause convergence problems. A model that directly includes the RPG effect (i.e., $\mathbf{a}_{2}=\mathbf{u}_{2}-\mathbf{Z g}$, where $\mathbf{u}_{2}$ are the GEBV of genotyped animals) requires only $m+n$ equations and has presumably better numerical properties. This was introduced by Mäntysaari and Strandén (2016) and has been tested by Vandenplas et al. (2019).

\section{SNP Augmented Models}

The ssHM (Fernando et al., 2016) and ssMEM approach by Mäntysaari and Strandén (2016) actually model the observations by SNP effects. In Liu et al. (2014), the model equation for the observations were the same as in AM BLUP or in ssGBLUP, but the difference was in the definition of variance structure of the breeding values. The blocks for the breeding values were as in $\mathbf{H}$, but after that, they augmented the random SNP effects into the vector of breeding values. Finally, they derived the inverse of the augmented variance-covariance matrix $\mathbf{H}_{a}$ :

$$
\mathbf{H}_{a}^{-1}=\left[\begin{array}{ccc}
\mathbf{A}^{11} & \mathbf{A}^{12} & 0 \\
\mathbf{A}^{21} & \mathbf{A}^{22}+\left(\frac{1}{w}-1\right) \mathbf{A}_{22}^{-1} & -\frac{1}{w} \mathbf{A}_{22}^{-1} \mathbf{Z} \\
0 & -\frac{1}{w} \mathbf{Z}^{\prime} \mathbf{A}_{22}^{-1} & \frac{1}{w} \mathbf{Z}^{\prime} \mathbf{A}_{22}^{-1} \mathbf{Z}+\mathbf{B}^{-1}
\end{array}\right] .
$$

In their notation, the matrix $\mathbf{B}$ was $\operatorname{var}(\mathbf{g}) / \operatorname{var}(\mathbf{a})$, and thereafter $\mathbf{B}$ has to be discounted by $(1-w)$, a proportion of genetic variance described by markers. After discounting, $\mathbf{B}^{-1}=\frac{m}{(1-w)} \mathbf{I}$. Note that the expression $\frac{1}{w}$ in $\mathbf{H}_{a}^{-1}$ requires that $w>0$.

The advantage of the ssMEM by Liu et al. (2014) is that $\mathbf{H}_{a}^{-1}$ is relatively easy to include in the existing MME, because the SNP effects are not actually in the statistical model equation. It also suggests a block iterative approach (hereafter called the Liu-Goddard approach), where SNP solutions can be computed using a simple update $\hat{\mathbf{g}}=\left(\mathbf{Z}^{\prime} \mathbf{A}_{22}^{-1} \mathbf{Z}+\mathbf{B}^{-1}\right)^{-1} \mathbf{Z}^{\prime} \mathbf{A}_{22}^{-1} \hat{\mathbf{u}}_{2}$, and thereafter the new single-step breeding values can be 
solved from an ordinary animal model in which the left-hand sides of MME for the genotyped animals are be corrected by $\hat{\gamma}=-\lambda \mathbf{A}_{22}^{-1}\left[(1-w) \hat{\mathbf{u}}_{2}-\mathbf{Z} \hat{\mathbf{g}}\right] / /$.

The stepwise block iteration in the Liu-Goddard approach was found to have very poor convergence (I. Strandén and E. A. Mäntysaari, Jokioinen, Finland, personal communication). This can be easily understood by considering the operation of such a block iterative algorithm with a very low $w$ value. It seems to be better to include the SNP as dummy effects in the MME and use PCG iteration directly in the full MME (Vandenplas et al., 2019). Another simplification is to absorb the SNP effect equations into the breeding values of genotyped animals in $\mathbf{u}_{2}$, which gives us single-step evaluations where the $\mathbf{G}^{-1}$ is replaced by computational formula based on the structure of $\mathbf{G}$ (see derivation in the Computational Solutions section) in the equations by Mäntysaari et al. (2017).

\section{Alternative Genomic Models}

A basic single-step SNP BLUP assumption is that all SNP can potentially have an effect, and all of them have the same a priori distribution. This assumption leads to equivalence of the random regression-based SNP BLUP and the genomic relationships-based GBLUP (Strandén and Garrick 2009).

From the very beginning, the ssMEM Fernando et al. (2014) and super hybrid model by Fernando et al. (2016) were built to allow any a priori assumptions for the SNP effects. Additionally, their computing implementations were based on Monte Carlo sampling, which yields not only the GEBV but also the posterior standard errors of estimates. Bayesian models or other variable selection models are becoming more useful along with increasing genotyping densities. Although no advantages have been found with high-density (700k) genotyping chips over the medium (54k) chips with GBLUP assumptions, more benefits are expected when SNP in the high-density data are given unequal weights. VanRaden et al. (2017) obtained significant improvement in validation reliability by adding 16,648 selected candidate variants from the imputed full sequences into standard BayesA-type SNP-BLUP evaluation with 61k SNP. Fragomeni et al. (2019) tested the same candidate variants as VanRaden et al. (2017), with a very similar nonlinear weighting strategy, in ssGBLUP but did not find any gain in validation accuracy. The way the nonlinear weights were applied was computationally tedious. After certain intervals of PCG iteration, the SNP effects were backsolved from the breeding value solutions, and individual SNP weights were derived from these SNP effect solutions; thereafter the new $\mathbf{G}$ matrix was built using different weights for the individual SNP.

It is easy to use unequal weights for the SNP in the ssMEM approaches. The models can be full Bayesian regressions or approximate nonlinear weights as in VanRaden (2008) and VanRaden et al. (2017). Moreover, the ssMEM approaches offer a straightforward way to include different SNP for different traits in a multitrait context. Theoretically, it could be also possible to define different correlations between traits for different SNP effects, although with current data sets the power to estimate such correlations might be limited.

\section{Single-Step Models with Meta-Founders}

In ssGBLUP, genotyped and ungenotyped animal breeding values should have the same expected values. This can be true only if the genomic and pedigree information are consistent. Several approaches have been proposed to align the $\mathbf{G}, \mathbf{A}$, and $\mathbf{A}_{22}$ matrices into the same scale (see discussion in Legarra et al., 2014). The first approaches attempted to scale the $\mathbf{G}$ matrix so that its diagonal in the base population would average 1.00 (VanRaden, 2008) or so that the traces of the $\mathbf{G}$ and $\mathbf{A}_{22}$ matrices would be the same (in Koivula et al. 2012a,b, 2015). Another, more appealing approach is to modify matrices $\mathbf{A}$ and $\mathbf{A}_{22}$ to be compatible with the G matrix. This was suggested by Christensen (2012) and formally presented with the so-called meta-founder concept by Legarra et al. (2015).

In the meta-founder approach, the founder animals are assumed to be members of appropriate base populations (Legarra et al., 2015). These base populations are formed from pools of independent gametes. The number of gametes determines the self-relatedness of the meta-founder population. For animals descending from this group, there is a nonzero probability of receiving the same gamete twice. When the 2 populations partly share the same gametes, the probabilities of drawing out the same gametes correspond to relationship among the populations. We can define base populations A and B with self-relatedness parameters $\gamma^{A}$ and $\gamma^{B}$ and relatedness $\gamma^{A B}$ across populations. When these parameters are known, the meta-founders can be considered similar to animals with a "relationship matrix" $\boldsymbol{\Gamma}$, and we can construct the relationship matrices $\mathbf{A}^{\Gamma}$ (or $\mathbf{A}_{22}^{\Gamma}$ ) with the tabular rules (Emik and Terrill, 1949). Alternatively, if the inverses $\mathbf{A}^{\Gamma^{-1}}$ or $\mathbf{A}_{22}^{\Gamma^{-1}}$ are needed, the rules of Henderson (1976) can be used with the inbreeding coefficients from the diagonals of $\mathbf{A}^{\Gamma}$. The $\boldsymbol{\Gamma}$ matrix can be estimated in several ways. The information comes from the known genotypes, from which it will be extrapolated to the base animals. The simplest approach is to 
estimate the base population allele frequencies and calculate $\boldsymbol{\Gamma}=8 \mathbf{P P}^{\prime}$, where the rows of $\mathbf{P}$ pertain to the meta-founder base populations, and the columns are the estimated allele frequencies for the SNP genotypes (Garcia-Baccino et al., 2017).

An advantage of the meta-founder approach is that also $\mathbf{A}^{\Gamma^{-1}}$ will become compatible with $\mathbf{G}^{-1}$ and not only the $\mathbf{A}_{22}^{\Gamma^{-1}}$ matrix. The meta-founder concept is very easy to understand, because it only replaces the unknown parent groups (UPG) with meta-founders, with "phantom parents" that are both "inbred" and related to each other. The biggest problem in its implementation is the definition of the $\boldsymbol{\Gamma}$ matrix. The number of base populations in current dairy cattle evaluations, defined by UPG, is typically counted in hundreds. It is simply not possible to estimate allele frequencies for every UPG counterpart, because some of them might not have any relationships with the genotyped animals.

\section{COMPUTATIONAL SOLUTIONS}

After the publication of the single-step methodology, the biggest hindrance to implementation was the computational cost. The need to invert 2 matrices the size of the number of genotyped animals suggested a definite limit on how many genotyped animals could be included in the evaluations. Thus, a major part of early research into the single-step approach was directed toward methods of avoiding these full inverses.

\section{Algorithm for Proven and Young}

Misztal et al. (2014) proposed to avoid computation of the full inverse of $\mathbf{G}$ with an approximation called the algorithm for proven and young (APY):

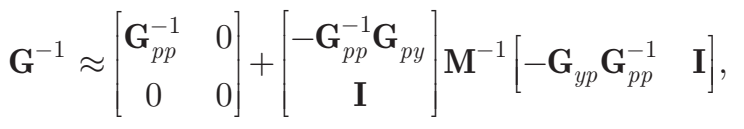

where the original $\mathbf{G}$ matrix is partitioned into blocks for proven animals $(p)$ and young animals $(y)$, and the $\mathbf{M}$ matrix is a diagonal matrix with elements

$$
m_{i i}=\left(\mathbf{G}_{y y}-\mathbf{G}_{y p} \mathbf{G}_{p p}^{-1} \mathbf{G}_{p y}\right)_{i i} .
$$

Since introduction of the APY, the original terms "proven" and "young" have been deemed misleading, and proven animals have been renamed core animals. When the number of genotyped animals increases, the number of computations used in forming APY $\mathbf{G}^{-1}$ increases linearly, as long as the number of core animals remains fixed. Consequently, APY is scalable even to large genotyped populations (Misztal 2016; Masuda et al., 2018).

A slightly simpler derivation of the APY approximation can be given via partial regression coefficients and Cholesky decomposition. Assuming that the genotyped animals have been arranged into blocks of core and young animals, as is also in Equation [3], the Cholesky decomposition can be written as follows:

$$
\mathbf{L}=\left[\begin{array}{cc}
\mathbf{L}_{c c} & 0 \\
\mathbf{L}_{y c} & \mathbf{L}_{y y}
\end{array}\right],
$$

where the part matrix $\mathbf{L}_{y y}$ is a triangular Cholesky factor of the (co)variance matrix of the young animals conditional on the core animals. In the Cholesky of $\mathbf{G}_{A P Y}$, this block is defined as a diagonal:

$$
\mathbf{L}_{A P Y}=\left[\begin{array}{lc}
\mathbf{L}_{c c} & 0 \\
\mathbf{L}_{y c} & \operatorname{diag}\left(\mathbf{G}_{y y}-\mathbf{L}_{y c} \mathbf{L}_{y c}{ }^{\prime}\right)
\end{array}\right],
$$

and the inverse of the matrix shown in Equation [3] is now

$$
\mathbf{G}_{A P Y}^{-1}=\mathbf{L}_{A P Y}^{-T} \mathbf{L}_{A P Y}^{-1} .
$$

Later Misztal (2016) and Pocrnic et al. (2016) interpreted the APY algorithm in detail. With the right choice of core animals, all the segregating haplotypes in the population can be described by core animal genotypes, and, thus, $\mathbf{G}_{A P Y}^{-1}$ can be a good approximation of $\mathbf{G}^{-1}$.

\section{Single-Step GTBLUP}

Columns in the genotype matrix can be centered and standardized so that $\mathbf{G}$ can be written simply as $\mathbf{G}=$ ZZ' (VanRaden 2008). After this, the columns are no longer independent. Moreover, when many more animals are genotyped than there are markers, the rank of $\mathbf{G}$ is much lower than its order. A common solution is to add a small regularization constant to the diagonal, or a small fraction ( $w$ ) of matrix $\mathbf{A}_{22}$ to the $\mathbf{Z} \mathbf{Z}^{\prime}$ matrix. We denote this regularization matrix as $\mathbf{C}$. The inverse of $\mathbf{G}$ can be written thus:

$$
\mathbf{G}_{C}^{-1}=\left(\mathbf{Z Z}^{\prime}+\mathbf{C}\right)^{-1}
$$

When $\mathbf{C}$ is $w \mathbf{A}^{22}$, we need to scale $\mathbf{Z Z}$ ' by the corresponding proportion $(1-w)$, but for simplicity we assume that during the standardization the columns in $\mathbf{Z}$ are multiplied by $\sqrt{1-w}$. Using the Woodbury matrix 
identity, the inverse $\mathbf{G}$ can be written with any form of C:

$$
\mathbf{G}_{C}^{-1}=\mathbf{C}^{-1}-\mathbf{C}^{-1} \mathbf{Z}\left(\mathbf{Z}^{\prime} \mathbf{C}^{-1} \mathbf{Z}+\mathbf{I}\right)^{-1} \mathbf{Z}^{\prime} \mathbf{C}^{-1} .
$$

When the regularization is only a constant added to the diagonal (i.e., $\mathbf{G}_{\varepsilon}=\mathbf{Z} \mathbf{Z}^{\prime}+\varepsilon \mathbf{I}$ ), the inverse has a simple form:

$$
\mathbf{G}_{\varepsilon}^{-1}=\frac{1}{\varepsilon} \mathbf{I}-\frac{1}{\varepsilon} \mathbf{Z}\left(\mathbf{Z}^{\prime} \mathbf{Z}+\varepsilon \mathbf{I}\right)^{-1} \mathbf{Z}^{\prime}
$$

Note that $\mathbf{Z}\left(\mathbf{Z}^{\prime} \mathbf{Z}+\varepsilon \mathbf{I}\right)^{-1} \mathbf{Z}^{\prime}$ is a projection matrix from a simple SNP-BLUP equation with $\varepsilon$ as a SNP variance.

When $\mathbf{Z}$ has many more rows than columns $\left(n_{q}>>\right.$ $m$ ), the computationally most expensive part of $\mathbf{G}_{\varepsilon}^{-1}$ is forming the $\mathbf{Z}^{\prime} \mathbf{Z}$ matrix. The matrix inverse in this form of $\mathbf{G}_{\varepsilon}^{-1}$ is only the inverse of the SNP-BLUP kernel, which is the size of the number of columns in $\mathbf{Z}$; that is, the number of SNP. In solving the MME with the PCG iteration, the key cost is a matrix-times-vector multiplication, in which a so-called direction vector is multiplied by the left-hand side of the MME. For $\mathbf{G}_{C}^{-1}$ in Equation [1], this means multiplying $\mathbf{G}_{C}^{-1} \mathbf{d}$.

Computationally the most economical form would be to factorize only the SNP-BLUP kernel as $\mathbf{Z}^{\prime} \mathbf{Z}+\varepsilon \mathbf{I}=$ $\mathbf{L} \mathbf{L}^{\prime}$ and, afterward, to store the matrices $\mathbf{Z}$ and $\mathbf{L}$ and perform all the matrix-times-vector multiplications and backsolves in sequence from the innermost parentheses outward:

$$
\mathbf{G}_{\varepsilon}^{-1} \mathbf{d}=\frac{1}{\varepsilon}\left(\mathbf{d}-\mathbf{Z}\left\{\mathbf{L} \backslash\left[\mathbf{L}^{\prime} \backslash\left(\mathbf{Z}^{\prime} \mathbf{d}\right)\right]\right\}\right),
$$

where the backslash $(\backslash)$ is an operator indicating solving the equation by forward or backward substitutions, without need for matrix inverses. Another alternative is to form a $\mathbf{T}$ matrix as $\mathbf{T}=\mathbf{L}^{-1} \mathbf{Z}$, which can, similarly to $\mathbf{G}_{A P Y}$, easily be used in existing evaluation software applications. Thus,

$$
\mathbf{G}_{\varepsilon}^{-1}=\frac{1}{\varepsilon} \mathbf{I}-\frac{1}{\varepsilon} \mathbf{T}^{\prime} \mathbf{T}
$$

Single-step GBLUP that are based on the T-matrix can be briefly called ssGTBLUP.

In case the $\mathbf{C}$ matrix is $w \mathbf{A}_{22}$, then

$$
\mathbf{G}_{w}=\mathbf{Z} \mathbf{Z}^{\prime}+w \mathbf{A}_{22}
$$

and

$$
\mathbf{G}_{w}^{-1}=\frac{1}{w} \mathbf{A}_{22}^{-1}-\frac{1}{w} \mathbf{T}_{w}{ }^{\prime} \mathbf{T}_{w}
$$

where $\mathbf{T}_{w}=\mathbf{L}_{w}^{-1} \mathbf{Z}^{\prime} \mathbf{A}_{22}^{-1}$ and $\mathbf{L}_{w}$ is the Cholesky factor, as in $\mathbf{Z}^{\prime} \mathbf{A}_{22}^{-1} \mathbf{Z}+w \mathbf{I}=\mathbf{L}_{w} \mathbf{L}_{w}^{\prime}$.

As already mentioned, the advantage of the $\mathbf{T}^{\prime} \mathbf{T}$ presentation is in avoiding the need to set up the $\mathbf{G}_{C}^{-1}$ matrix explicitly. Because in the iterative solving via the PCG method, the $\mathbf{G}_{C}^{-1}$ matrix is needed only in a left multiplication of a direction vector, the multiplication can be performed in sequence. And because the basic single-step equations involve the multiplication $\mathbf{A}_{22}^{-1} \mathbf{d}$, this multiplication can be performed only once, as $\left(\frac{1}{w}-1\right) \mathbf{A}_{22}^{-1} \mathbf{d}$. During the PCG iteration, the number of computational flops is directly relative to the number of nonzero elements in the external $\mathbf{T}$ matrix. If the evaluation includes $n_{q}$ genotyped animals with $\mathbf{G}_{C}$ defined using $m$ markers, and no rank reduction in $\mathbf{L} \mathbf{L}^{\prime}$ is wanted, the $\mathbf{T}$ matrix is an $m$-by- $n_{g}$ matrix, and the multiplication $\mathbf{T}^{\prime} \mathbf{T} \mathbf{d}$ in each PCG iteration round requires $2 n_{g} m$ flops. The corresponding cost in the APY algorithm is approximately $2 n_{g} n_{c}-n_{c}^{2}$, where $n_{c}$ is the number of core animals. If both approaches have the same fit (i.e., $n_{c}=m$ ) the computational cost becomes almost the same as when $n_{g}>>m$; that is, when there are many more genotyped animals than the assumed rank of $\mathbf{Z}$.

\section{Sparse Presentation of $\mathrm{A}_{22}^{-1}$ Matrix}

The $\mathbf{H}$ matrix of the original ssGBLUP [1] also includes matrices $\mathbf{A}^{-1}$ and $\mathbf{A}_{22}^{-1}$. If the computation is based on an approach where the $\mathbf{G}$ matrix is explicitly formed in full, it is practical also to form $\mathbf{A}_{22}^{-1}$ in full and subtract it from $\mathbf{G}^{-1}$. However, when the $\mathbf{G}$ matrix presentation is sparse like in $\mathbf{G}_{A P Y}$ or when the matrix multiplication $\mathbf{G}^{-1} \mathbf{d}$ is implicit like in ssGTBLUP, all savings are lost unless $\mathbf{A}_{22}^{-1}$ is also treated as a matrix product with sparse factors. A simple block matrix inverse results:

$$
\mathbf{A}_{22}^{-1}=\mathbf{A}^{22}-\mathbf{A}^{21}\left(\mathbf{A}^{11}\right)^{-1} \mathbf{A}^{12},
$$

where $\mathbf{A}^{22}, \mathbf{A}^{21}, \mathbf{A}^{11}$, and $\mathbf{A}^{12}$ are sparse submatrices of $\mathbf{A}^{-1}$. Thus, the multiplication $\mathbf{A}_{22}^{-1} \mathbf{d}$ can be performed in sequence:

$$
\mathbf{A}_{22}^{-1} \mathbf{d}=\mathbf{A}^{22} \mathbf{d}-\mathbf{A}^{21}\left(\mathbf{A}^{11}\right)^{-1} \mathbf{A}^{12} \mathbf{d}
$$


Surprisingly, all the matrix multiplications above can be performed more rapidly using only the pedigree file instead of setting up the matrices. The multiplication by $\left(\mathbf{A}^{11}\right)^{-1}$ is an exception, but it can be avoided because $\mathbf{A}^{11}$ is very sparse, and it can be efficiently factorized in sparse form (Strandén et al., 2017). Note also that only the ancestors of the genotyped animals can contribute to coefficients in $\mathbf{A}_{22}$, and thus it is unnecessary to include non-ancestors in the matrix multiplications $\mathbf{A}^{12} \mathbf{d}$ or to the factorized $\mathbf{A}^{11}$.

In Equation [5], a fraction $w$ of $\mathbf{A}_{22}$ was added to the $\mathbf{Z Z}^{\prime}$ matrix to ensure that the genomic relationship matrix is invertible. When the $w$ fraction is increased in an attempt to improve the model, the process can also be justified as model tuning or parametrization. Higher values of $w$ often improve the prediction properties of the model. With ssGTBLUP accounting, any level of $w$ is simple because it only results in a small rescaling of $\mathbf{T}_{w}$, and a change of the factor $\left(\frac{1}{w}-1\right)$ in multiplying $\mathbf{A}_{22}^{-1} \mathbf{d}$ during the iteration. In the APY algorithm, higher values of $w$ lead to higher degrees of approximation, because APY ignores those unconditional relationships among the young animals that are not described by their relationships to the core animals.

\section{IMPLEMENTATION ISSUES}

\section{Example of Single-Step Computation Costs}

We illustrate computational costs of single-step APY GBLUP and ssGTBLUP approaches with a large field data set from the Irish Beef Data and Genomics Programme. The model included maternal effects for all 6 analyzed calving traits (Strandén et al., 2019). There were 10.4 million animals in pedigree, of which 3.2 million had observations. The number of genotyped animals was 1.51 million, which all had genotypes for 50,955 SNP. At preprocessing, the eigenvalues of $\left(\mathbf{Z}^{\prime} \mathbf{Z}\right.$ $+\varepsilon \mathbf{I})$ were estimated, and only a desired proportion of variation in the $\mathbf{G}$ matrix was used in the analyses. Table 1 presents the computational costs of evaluations for a case where $98 \%$ of the variation in the $\mathbf{G}$ matrix was accounted for. This corresponded with 31,443 rows in the $\mathbf{T}$ matrix, and, for the comparison, the same number of animals was randomly chosen for the core in the APY algorithm. Convergence of PCG was assumed when $C_{r} \leq 10^{-7}$, with $C_{r}$ defined as a Frobenius norm of difference between the true right-hand side of the MME and the one predicted by the current solutions relative to the true right-hand side norm. The implementation was based on use of the Intel MKL parallel math library (Intel, 2015). All runs were given the opportunity to use $10 \mathrm{CPU}$ cores, and the timings in Table 1 are all wall clock times and therefore only rough estimates.

In this evaluation, the computing times for the single-step models were about 10 times that of the basic animal model. However, this time increase is very dependent on the model and the ratio of genotyped animals versus the number of observations. In the Irish beef data, almost $15 \%$ of the animals were genotyped. In another example, we illustrate the computing costs of test-day animal model and the same model including 178,000 genotyped animals in ssGTBLUP (Table 2 ). The basic model was the official Nordic Holstein production trait evaluation (Lidauer et al., 2015), with 9 traits and 42 random regression coefficients for each animal with records. The proportion of genotyped animals to the number of animals with records was only $2 \%$, and the solver computing time for ssGTBLUP was only $15 \%$ more than with AM BLUP (Table 2).

The computing time differences between the singlestep approaches in Table 1 were negligible. In both approaches, the convergence criteria $C_{r}$ fluctuated greatly, and it was only a matter of chance which one happened to reach the $10^{-7}$ limit first. Moreover, the used program code included calls on parallel MKL subroutine GEMM, and the time per iteration very much depended on the algorithm's suitability for such straightforward implementation. However, the time used in preprocessing to form the $\mathbf{T}$ matrix was more than the time needed for the APY $\mathbf{G}$ inverse. Some of this was because of the rank reduction, which required eigenvalue decomposition, but even excluding that, the ssGTBLUP preprocessing took more time.

Table 1. Computing time and memory use for animal model (AM) BLUP, single-step (ss)APY genomic BLUP (GBLUP), and ssGTBLUP; evaluations were for 6 calving traits in the Irish Beef Data and Genomics Programme (BDGP) with 10.4 million animals and 1.51 million genotyped animals (Strandén et al., 2019)

\begin{tabular}{lccc}
\hline Item & $\begin{array}{c}\text { AM } \\
\text { BLUP }\end{array}$ & $\begin{array}{c}\text { ssAPY } \\
\text { GBLUP }^{1}\end{array}$ & ssGTBLUP $^{2}$ \\
\hline Preprocessing & & & \\
$\quad$ Time (h) & & 7.7 & 10.3 \\
Peak memory (GB) & & 558 & 374 \\
Solving & 479 & 897 & 710 \\
$\quad$ Iterations & 9 & 55 & 51 \\
Seconds/PCG round & 1.3 & 14.4 & 12.2 \\
Time total (h) & 4 & 366 & 366 \\
Peak memory (GB) & &
\end{tabular}

${ }^{1}$ Single-step evaluations with APY (algorithm for proven and young) inverse of $\mathbf{G}$ (the matrix of genomic relationships).

${ }^{2}$ Single-step evaluations with implicit presentation of inverse of $\mathbf{G}$.

${ }^{3} \mathrm{PCG}=$ preconditioned conjugate gradient. 


\section{Convergence of Iterative Methods}

Single-step evaluations undoubtedly have higher computational costs compared with the current animal model evaluations. Also, with complicated multi-trait models and low heritable traits, single-step evaluations tend to require more iterations. Substantial efforts have been devoted to improving the convergence of iterative methods applied to single-step models. The PCG iteration is one of the most effective iterative algorithms, but it is also very sensitive to dependencies within the model effects and to a poor condition number of coefficient matrix. In our test runs, the single most important finding was the importance of the balance between pedigree relationships of genotyped animals $\left(\mathbf{A}_{22}^{-1}\right)$, genomic relationships in $\mathbf{G}^{-1}$, and genetic groups. When the genetic groups were included in the model as regression effects of UPG contributions, the convergence was always satisfactory (Strandén et al., 2019). This was the way groups were treated in the Irish beef evaluation (Table 1). But when UPG were included in $\mathbf{A}^{-1}$ as "phantom parents," using simple rules described by Quaas (1988), the convergence was slow and sometimes unachievable (Matilainen et al., 2018). The problem seemed to be worse with problematic traits such as fertility, with very low heritabilities and high correlations among traits. Our conclusion was that UPG must be treated as co-variables, or, when more plausible, UPG effects can be changed to be "phantom parents" by applying the QP transformation (Westell et al., 1988) to complete the $\mathbf{H}^{-1}$ matrix. In practice, this means that for each genetic group, the elements in the

Table 2. Computing costs for animal model (AM) BLUP and singlestep (ss)GTBLUP in Nordic (DFS) production evaluations ${ }^{1}$

\begin{tabular}{lcc}
\hline Item & AM BLUP & ssGTBLUP $^{2}$ \\
\hline Preprocessing & & 12.9 \\
$\quad$ Time (h) & & 377 \\
Peak memory (GB) & & \\
Solving & 1,505 & 1,416 \\
Iterations & 138 & 163 \\
Seconds/PCG round & 57.5 & 66.5 \\
Time total (h) & 13.6 & 80.1 \\
Peak memory (GB) & & \\
\hline
\end{tabular}

${ }^{1}$ Holstein test-day model evaluations with 8.3 million animals with records and 178,000 genotyped animals (Koivula, unpublished data). The evaluations accounted for inbreeding coefficients in $\mathbf{A}^{-1}$, unknown parent groups by the QP transformation of $\mathbf{H}^{-1}$, and diagonal preconditioner, also including estimates of diagonal of $\mathbf{A}_{22}^{-1}$. $\mathbf{A}=$ the pedigreebased additive relationship matrix; $\mathbf{A}_{22}=$ part of the $\mathbf{A}$ matrix for the genotyped animals; $\mathbf{H}=$ unified relationship matrix. Convergence was assumed when $C_{r}<10^{-7}$ (the norm of relative error in the predicted right-hand side of mixed-model equations).

${ }^{2}$ Single-step evaluations with implicit presentation of the inverse of $\mathbf{G}$. ${ }^{3} \mathrm{PCG}=$ preconditioned conjugate gradient. row of $\mathbf{H}^{-1}$ corresponding to an animal are weighted by the proportions of genes the animals received from the group. The sum of these products is the contribution of the animal to the group. With the original $\mathbf{A}^{-1}$ matrix, this sum is 0 for all the animals with known parents, but after $\left(\mathbf{G}^{-1}-\mathbf{A}_{22}^{-1}\right)$ is added to matrix $\mathbf{A}^{-1}$, the group contribution sum will reflect the incompatibilities between $\mathbf{G}^{-1}$ and $\mathbf{A}_{22}^{-1}$.

The precondition matrix is critical to good convergence in the PCG iteration. It has no direct effect on the solutions, but it greatly affects computing time. The new algorithms that specify new computational forms of inverses of $\mathbf{G}$ and $\mathbf{A}_{22}$ matrices do not give information for the preconditioner straightforwardly, and sometimes the necessary values must be estimated separately. With the use of diagonal preconditioning, for example, the diagonals of $\mathbf{A}_{22}^{-1}$ might have to be computed separately. Similarly, when UPG are included using the QP transformation, the precondition matrix must be updated accordingly. Generally, convergence in iteration is better in the GBLUP models than in the SNP-BLUP models, and this seems to apply also to single-step evaluations with marker effects (Strandén and Mäntysaari, 2014). The equations specific to the SNP have very high off-diagonal elements, which presumable leads to poor (i.e., high) condition numbers of the coefficient matrix. This problem can be solved by using either so-called deflated preconditioning or 2 different preconditioning matrices (Vandenplas et al., 2019). The most extreme ssMEM model preconditioner has been proposed by Harris et al. (2019). In their implementation, the whole block of equations for the marker effects in Equation [2] is included in the preconditioner, and similarly the entire submatrices $\mathbf{W}_{1}{ }^{\prime} \mathbf{R}^{-1} \mathbf{W}_{1}+\lambda \mathbf{A}^{11}$ and $\mathbf{W}^{\prime} \mathbf{R}^{-1} \mathbf{W}+(1-w) \lambda \mathbf{A}^{-1}$ are also explicitly treated as part of the preconditioner.

\section{Bias in Single-Step Evaluations}

The models in dairy cattle genetic evaluations have been validated for a long time using so-called Interbull validation tests (Boichard et al., 1995) to test the consistency of genetic trends. When genomic evaluations were introduced, prediction accuracy also became of interest. VanRaden (2008) proposed to estimate the realized reliabilities of genomic evaluations using forward prediction. This was performed retrospectively by first deleting 4 years of data and then estimating the predictive power of the reduced-data bull evaluations in predicting daughter performance in the current data. The preferred daughter performances would be daughter yield deviations but also deregressed genetic evaluations (Mrode 2014). 
In addition to validation reliability, forward prediction also gives estimates of bias and over- or underdispersion (Mäntysaari et al., 2010). Bias in EBV can be defined as a mean in difference between estimated breeding values and modeled phenotypic performances. Overdispersion is measured via the regression coefficient of the modeled phenotypes on the reduced data breeding value estimates. Overdispersion has commonly been called bias, which can be justified because, in selected traits, bias and dispersion are related. If young animals (bulls or cows) have a selection difference, such as $100 \mathrm{~kg}$, of which only $80 \%$ will be realized (i.e., overdispersion $b_{1}=0.80$ ), then the bias, as a difference between the predicted performance and the observed phenotypes, is about $20 \mathrm{~kg}$. Thus, when the genomic evaluation overpredicts the differences among animals, it also overpredicts the values of the selected animals.

Genomic models seem to have a general bias problem, at least with selected traits. Bias in dairy cattle single-step evaluations has been estimated in several studies (Koivula et al., 2015, 2018; Oliviera et al., 2019; Tsuruta et al., 2019). Table 3 illustrates the validation test results in Nordic Holstein single-step test-day evaluations described in Table 2. The model was a multi-trait, multi-lactation random regression model with 15 breeding value coefficients per animal (Lidauer et al., 2015). The validation reliabilities are somewhat lower than in the official Nordic (DFS) genomic evaluations, because here only data from Denmark, Finland, and Sweden were considered, whereas the official evaluations include genotypes and MACE EBV of bulls from 6 EuroGenomics Consortium countries. The overdispersions $\left(b_{1}\right)$ are $0.80,0.72$, and 0.73 for milk, protein, and fat, respectively. If GEBV were submitted to Interbull as an input for the genomic MACE, either the values should be higher than 0.90 or their $95 \%$ confidence limit should include 1 .
In early studies, the bias in single-step evaluations were fine-tuned using scaling parameters $\lambda, \tau$, and $\omega$ in $\mathbf{H}^{-1}$ (Misztal 2017):

$$
\mathbf{H}^{-1}=\mathbf{A}^{-1}+\left[\begin{array}{cc}
0 & 0 \\
0 & \lambda\left(\tau \mathbf{G}_{w}^{-1}-\omega \mathbf{A}_{22}^{-1}\right)
\end{array}\right] .
$$

The factors $w$ and $\lambda$ simply determine the weight for genomic information versus weight for pedigree, the former in direct scale and the latter in inverse scale. The parameters $\tau$ and $\omega$ adjust the scale differences in matrices $\mathbf{G}_{w}$ and $\mathbf{A}_{22}$ (see discussion in Tsuruta et al., 2019). When genotyped animals have missing parents, inbreeding in $\mathbf{A}_{22}$ is underestimated, which can then be partly patched by using $\omega<\tau$. Generally, bias can be controlled through adjustments (Koivula et al., 2015; Oliviera et al., 2019), especially by $w$ and $\omega$, but higher values of RPG $(w>0.4)$ and low values of $\omega(<0.8)$ can start to show through reduced validation reliability and lower genetic trend. In multi-step evaluations, more tools exist for adjusting the bias. This is because the adjustments, such as use of lower heritability or scaling the GEBV variance according to validation accuracy, do not affect the official national EBV.

The "Interbull standard" validation test (Mäntysaari et al., 2010) itself has been criticized for not functioning correctly in genomic selected data. The test was introduced when genotyped bulls were selected to be used in AI by their parent average EBV. Currently, bull calves are selected by their GEBV, which automatically reduces the expected validation reliability $\mathrm{R}^{2}$. Moreover, the bias is quantified in a scale of deregressed genetic evaluations from the ordinary pedigree-based evaluations. When bulls are preselected using the GEBV, the subsequently calculated animal model EBV will be downward biased (Mäntysaari et al., 2018), and thus,

Table 3. Validation results for Nordic Holstein single-step test-day evaluations ${ }^{1}$

\begin{tabular}{llccl}
\hline Item & Evaluation & $\operatorname{mean}\left[(\mathrm{G}) \mathrm{EBV}_{\mathrm{r}}-\mathrm{DRP}\right]$ & $b_{1}{ }^{1}$ & $\mathrm{R}_{v}{ }^{22}$ \\
\hline Milk & PA-EBV & 182.92 & 0.72 & 0.20 \\
& ssGEBV & 435.34 & 0.80 & 0.55 \\
Protein & PA-EBV & 2.70 & 0.63 & 0.16 \\
& ssGEBV & 16.14 & 0.72 & 0.45 \\
Fat & PA-EBV & 5.48 & 0.69 & 0.20 \\
& ssGEBV & 20.51 & 0.73 & 0.49 \\
\hline
\end{tabular}

${ }^{1}$ Results are from 643 validation bulls that had no daughters in data with last 4 years removed but had recent deregressed evaluations (DRP) with $>3$ effective records (equivalent to 20 daughters). Single-step genomic enhanced breeding values (ssGEBV) evaluations included $30 \%$ residual polygenic effect (Koivula et al., unpublished data). $\mathrm{PA}-\mathrm{EBV}=$ bull parent average EBV, computed from reduced data

${ }^{1}$ Validation regression DRP $=b_{0}+b_{1}(\mathrm{G}) \mathrm{EBV}$, weighted by DRP reliability, where GEBV and EBV were the single-step and animal model BLUP evaluations from reduced data, and $b_{0}$ and $b_{1}$ are the regression coefficients describing the model bias.

${ }^{2}$ Validation reliability $=\mathrm{R}^{2} / \bar{r}$, where $\mathrm{R}^{2}$ is coefficient of determination from the regression, and $\bar{r}$ is average weight (i.e., the mean of DRP reliabilities). 
genomic selection does not seem to be realized. This is clear in the Table 3 values of bias. When deregressed evaluations are subtracted from the EBV, the result indicates that selection has not been expressed fully in bulls entering service during the last 4 years.

Legarra and Reverter (2018) proposed to estimate bias as the difference between current evaluations (named $\mathrm{EBV}_{\text {whole }}$ ) and reduced evaluations (named EB$\mathrm{V}_{\text {partial }}$ ) and overdispersion using the regression of EB$\mathrm{V}_{\text {whole }}$ on $\mathrm{EBV}_{\text {partial }}$. In validation of genomic evaluations, GEBV would be used in place of EBV. They called the method LR. Although the LR method does not directly give any estimates of validation reliability, it would give the relative increase in reliability $R_{E B \text { Vwhole }}^{2} / R_{E B \text { Vpartial }}^{2}$ as a reciprocal of the correlation between $\mathrm{EBV}_{\text {whole }}$ and $\mathrm{EBV}_{\text {partial }}$.

\section{SUMMARY}

Genomic evaluation principles were introduced more than 10 years ago, and, currently, genomic selection is the main source of genetic improvement in dairy cattle worldwide. Because this selection cannot be attributed to selection in cow records or in bull daughter averages, it will not be captured by the pedigree-based genetic evaluations. Therefore EBV are starting to become more and more biased. Currently bulls born in 2015 are getting their first progeny test results. These bulls and also their sires were preselected based on genomic evaluations. To ensure unbiased evaluations in the future, the only choice is to start including genomic information into national evaluations. This can be done using single-step evaluation approaches.

During the past decade, genotyping has become an everyday tool in the selection of female replacements as well. Many countries have also genotyped females with the aim of increasing the reference population for genomic evaluations. The ultimate goal might be to genotype all animals. Meanwhile national genetic evaluations have to be able to handle all non-genotyped animals as well as the progressively increasing number of genotyped animals. At the time of introduction of single-step genomic evaluation models, there was a fear that the applications would have an upper bound on how many genotypes can be included in the evaluations. With the current single-step techniques, the computing cost has become linear in the number of animals genotyped; thus, the algorithms are able to handle any size of dairy cattle populations. Single-step models with genomic relationships can be solved with ssGTBLUP (Mäntysaari et al., 2017) or single-step APY GBLUP (Misztal et al., 2014). Alternatively, computing can be performed using various marker effect-based models.
The most promising marker model approaches are hybrid ssHM (Fernando et al., 2016) and, if the convergence proves satisfactory, the models with augmented SNP effects and RPG (Liu et al., 2014).

When the genomic model is based on the Gaussian assumption that all markers have the same a priori variance, differences in the $\mathbf{G}$-based and marker-based single-step models are not large, especially if a residual polygenic effect is assumed and fitted. If in the future it becomes useful to give different weights for different SNP effects, or to fit different SNP for different traits in multi-trait models, ssMEM models have a clear advantage over methods that rely on the genomic relationship matrix $\mathbf{G}$.

Single-step evaluations are accused of overpredicting the differences in breeding values. Whereas overprediction of some animals is easy to correct in multi-step evaluations, single-step evaluations are presumed to be the only evaluations conducted in the population. For successful operation of breeding programs, the evaluations should rank the animals fairly, young and old, with or without genomic information, and with or without phenotypic information. Overprediction or overdispersion are most severe for the key traits in selection goals. Unfortunately those are also the traits in which single-step evaluations should most urgently be implemented. The bias is being studied intensively, and, hopefully, the universal solution for "discounting" genomic information will be found.

\section{ACKNOWLEDGMENTS}

Data used in the examples came from the Nordic Cattle Genetic Evaluation NAV (Aarhus, Denmark), from Viking Genetics (Randers, Denmark), and from the Irish Cattle Breeding Federation (Bandon, Ireland). Their input and support are greatly appreciated. We also thank the 2 anonymous reviewers for their helpful comments. The authors have not stated any conflicts of interest.

\section{REFERENCES}

Aguilar, I., I. Misztal, D. L. Johnson, A. Legarra, S. Tsuruta, and T. J. Lawlor. 2010. Hot topic: A unified approach to utilize phenotypic, full pedigree, and genomic information for genetic evaluation of Holstein final score. J. Dairy Sci. 93:743-752. https://doi.org/10 .3168/jds.2009-2730.

Boichard, D., B. Bonaiti, A. Barbat, and S. Mattalia. 1995. Three methods to validate the estimation of genetic trend for dairy cattle. J. Dairy Sci. 78:431-437. https://doi.org/10.3168/jds.S0022 -0302(95)76652-8.

Christensen, O. F. 2012. Compatibility of pedigree-based and marker-based relationship matrices for single-step genetic evaluation. Genet. Sel. Evol. 44:37. https://doi.org/10.1186/1297-9686-44-37.

Christensen, O. F., and M. S. Lund. 2009. Genomic relationship matrix when some animals are not genotyped. Page 299 in Proc. 
60th Annual Meeting European Association for Animal Production, Barcelona, Spain. Wageningen Press, Wageningen, the Netherlands.

Christensen, O. F., and M. S. Lund. 2010. Genomic prediction when some animals are not genotyped. Genet. Sel. Evol. 42:2. https:// doi.org/10.1186/1297-9686-42-2.

Colinet, F. G., J. Vandenplas, P. Faux, S. Vanderick, R. Renaville, C. Bertozzi, X. Hubin, and N. Gengler. 2013. Walloon single-step genomic evaluation system integrating local and MACE EBV. Interbull Bull. 47:203-210.

Emik, L. O., and C. E. Terrill. 1949. Systematic procedures for calculating inbreeding coefficients. J. Hered. 40:51-55. https://doi.org/ 10.1093/oxfordjournals.jhered.a105986.

Fernando, R. L., H. Cheng, B. L. Golden, and D. J. Garrick. 2016. Computational strategies for alternative single-step Bayesian regression models with large numbers of genotyped and non-genotyped animals. Genet. Sel. Evol. 48:96. https://doi.org/10.1186/ s12711-016-0273-2.

Fernando, R. L., J. C. Dekkers, and D. J. Garrick. 2014. A class of Bayesian methods to combine large numbers of genotyped and non-genotyped animals for whole-genome analyses. Genet. Sel. Evol. 46:50. https://doi.org/10.1186/1297-9686-46-50.

Fernando, R. L., D. Garrick, and J. C. M. Dekkers. 2013. Bayesian regression method for genomic analyses with incomplete genotype data. Pages 26-30 in Proc. 64th Annual Meeting of the European Federation of Animal Science, August 2013, Nantes, France, Vol. 225 .

Fragomeni, B. O., D. A. L. Lourenco, A. Legarra, P. M. VanRaden, and I. Misztal. 2019. Alternative SNP weighting for single-step genomic best linear unbiased predictor evaluation of stature in US Holsteins in the presence of selected sequence variants. J. Dairy Sci. 102:10012-10019. https://doi.org/10.3168/jds.2019-16262.

Garcia-Baccino, C. A., A. Legarra, O. F. Christensen, I. Misztal, I. Pocrnic, Z. G. Vitezica, and R. J. Cantet. 2017. Metafounders are related to $F_{\text {st }}$ fixation indices and reduce bias in single-step genomic evaluations. Genet. Sel. Evol. 49:34. https://doi.org/10 .1186/s12711-017-0309-2.

Gengler, N., P. Mayeres, and M. Szydlowski. 2007. A simple method to approximate gene content in large pedigree populations: Application to the myostatin gene in dual-purpose Belgian Blue cattle. Animal 1:21-28. https://doi.org/10.1017/S1751731107392628.

Goddard, M. E., B. J. Hayes, and T. H. Meuwissen. 2011. Using the genomic relationship matrix to predict the accuracy of genomic selection. J. Anim. Breed. Genet. 128:409-421. https://doi.org/10 .1111/j.1439-0388.2011.00964.x.

Gonzalez-Peña, D., N. Vukasinovic, J. J. Brooker, C. A. Przybyla, and S. K. DeNise. 2019. Genomic evaluation for calf wellness traits in Holstein cattle. J. Dairy Sci. 102:2319-2329. https://doi.org/10 .3168/jds.2018-15540.

Harris, B. L., R. G. Sherlock and A. M. Winkelman. 2019. Marker model for genomic prediction in the Livestock Improvement breeding scheme. LIC Technical Document, November 2018. Updated Feb. 28, 2019.

Henderson, C. R. 1976. A simple method for computing the inverse of a numerator relationship matrix used in prediction of breeding values. Biometrics 32:69-83. https://doi.org/10.2307/2529339.

Henderson, C. R. 1984. Applications of Linear Models in Animal Breeding (Vol. 462). University of Guelph, Guelph, Canada.

Intel. 2015. Intel Math Kernel Library reference manual. Accessed Mar. 2, 2020. https://software.intel.com/en-us/mkl-reference -manual-for-fortran.

Interbull. 2019. National genomic evaluation forms provided by countries: Genoforms. Accessed Jun. 17, 2019. https://interbull.org/ib/ nationalgenoforms.

Koivula, M., I. Strandén, G. P. Aamand, and E. A. Mäntysaari. 2018. Reducing bias in the dairy cattle single-step genomic evaluation by ignoring bulls without progeny. J. Anim. Breed. Genet. 135:107115. https://doi.org/10.1111/jbg.12318.

Koivula, M., I. Strandén, J. Pösö, G. P. Aamand, and E. A. Mäntysaari. 2012b. Single step genomic evaluations for the Nordic Red
Dairy Cattle test day data. Pages 115-120 in Proc. 2012 Interbull meeting, Cork, Ireland.

Koivula, M., I. Strandén, J. Pösö, G. P. Aamand, and E. A. Mäntysaari. 2015. Single-step genomic evaluation using multitrait random regression model and test-day data. J. Dairy Sci. 98:2775-2784. https://doi.org/10.3168/jds.2014-8975.

Koivula, M., I. Strandén, G. Su, and E. A. Mäntysaari. 2012a. Different methods to calculate genomic predictions-Comparisons of BLUP at the single nucleotide polymorphism level (SNP-BLUP), BLUP at the individual level (G-BLUP), and the one-step approach (H-BLUP). J. Dairy Sci. 95:4065-4073. https://doi.org/10 .3168/jds.2011-4874.

Legarra, A., O. F. Christensen, I. Aguilar, and I. Misztal. 2014. Single step, a general approach for genomic selection. Livest. Sci. 166:5465. https://doi.org/10.1016/j.livsci.2014.04.029.

Legarra, A., O. F. Christensen, Z. G. Vitezica, I. Aguilar, and I. Misztal. 2015. Ancestral relationships using metafounders: Finite ancestral populations and across population relationships. Genetics 200:455-468. https://doi.org/10.1534/genetics.115.177014.

Legarra, A., and V. Ducrocq. 2012. Computational strategies for national integration of phenotypic, genomic, and pedigree data in a single-step best linear unbiased prediction. J. Dairy Sci. 95:46294645. https://doi.org/10.3168/jds.2011-4982.

Legarra, A., and A. Reverter. 2018. Semi-parametric estimates of population accuracy and bias of predictions of breeding values and future phenotypes using the LR method. Genet. Sel. Evol. 50:53. https://doi.org/10.1186/s12711-018-0426-6.

Lidauer, M. H., J. Pösö, J. Pedersen, J. Lassen, P. Madsen, E. A. Mäntysaari, U. S. Nielsen, J.-Å. Eriksson, K. Johansson, T. Pitkänen, I. Strandén, and G. P. Aamand. 2015. Across-country test-day model evaluations for Holstein, Nordic Red Cattle, and Jersey. J. Dairy Sci. 98:1296-1309. https://doi.org/10.3168/jds.2014-8307.

Liu, Z., M. E. Goddard, F. Reinhardt, and R. Reents. 2014. A singlestep genomic model with direct estimation of marker effects. J. Dairy Sci. 97:5833-5850. https://doi.org/10.3168/jds.2014-7924.

Lourenco, D. A. L., S. Tsuruta, B. O. Fragomeni, Y. Masuda, I. Aguilar, A. Legarra, S. Miller, D. Moser, and I. Misztal. 2018. Singlestep genomic BLUP for national beef cattle evaluation in US: From initial developments to final implementation. Page 495 in Proc. 11th World Congr. Genetics Appl. Livest. Prod., Auckland, New Zealand.

Malchiodi, F., J. Jamrozik, A. M. Christen, G. Kistemaker, P. Sullivan, B. Van Doormaal, D. Kelton, F. Schenkel, and F. Miglior. 2018. Implementation of genomic evaluation for digital dermatitis in Canada. Interbull Bull. 53:71-75.

Mäntysaari, E. A., G. P. Aamand, I. Strandén, and M. Koivula. 2018. Solutions for the fixed effects, yield deviations and daughter yield deviations from a data subject to genomic selection. Interbull Open Meeting, Auckland, New Zealand. http://interbull.org/ static/web/1500EsaMäntysaari.pdf.

Mäntysaari, E. A., R. D. Evans, and I. Strandén. 2017. Efficient single-step genomic evaluation for a multibreed beef cattle population having many genotyped animals. J. Anim. Sci. 95:4728-4737. https://doi.org/10.2527/jas2017.1912.

Mäntysaari, E. A., M. Koivula, I. Strandén, J. Pösö, and G. P. Aamand. 2011. Estimation of GEBVs using deregressed individual cow breeding values. Interbull Bull. 44:19-24.

Mäntysaari, E. A., Z. Liu, and P. VanRaden. 2010. Interbull validation test for genomic evaluations. Proceedings of the Interbull industry meeting "Genomic information in genetic evaluations," Paris, France. Interbull Bull. 41:17.

Mäntysaari, E. A., and I. Strandén. 2016. Single-step genomic evaluation with many more genotyped animals. In Proc. 67th Annual Meeting of the European Association for Animal Production, Belfast, Northern Ireland.

Masuda, Y., P. M. VanRaden, I. Misztal, and T. J. Lawlor. 2018. Differing genetic trend estimates from traditional and genomic evaluations of genotyped animals as evidence of preselection bias in US Holsteins. J. Dairy Sci. 101:5194-5206. https://doi.org/10.3168/ jds.2017-13310. 
Matilainen, K., I. Strandén, G. P. Aamand, and E. A. Mäntysaari. 2018. Single step genomic evaluation for female fertility in Nordic Red dairy cattle. J. Anim. Breed. Genet. 135:337-348. https://doi .org/10.1111/jbg. 12353.

Misztal, I. 2016. Inexpensive computation of the inverse of the genomic relationship matrix in populations with small effective population size. Genetics 202:401-409. https://doi.org/10.1534/genetics.115 .182089 .

Misztal, I. 2017. Studies on inflation of GEBV in single-step GBLUP for type. Proceedings of the Interbull Meeting, Tallinn, Estonia. Interbull Bull. 51.

Misztal, I., Z. G. Vitezica, A. Legarra, I. Aguilar, and A. A. Swan. 2013. Unknown-parent groups in single-step genomic evaluation. J. Anim. Breed. Genet. 130:252-258. https://doi.org/10.1111/jbg .12025 .

Misztal, I., A. Legarra, and I. Aguilar. 2014. Using recursion to compute the inverse of the genomic relationship matrix. J. Dairy Sci. 97:3943-3952. https://doi.org/10.3168/jds.2013-7752.

Mrode, R. A. 2014. Linear Models for the Prediction of Animal Breeding Values. 3rd ed. Cabi International, Wallingford, UK.

Oliveira, H. R., D. A. L. Lourenco, Y. Masuda, I. Misztal, S. Tsuruta, J. Jamrozik, J. L. F. Brito, F. F. Silva, and F. S. Schenkel. 2019. Application of single-step genomic evaluation using multiple-trait random regression test-day models in dairy cattle. J. Dairy Sci. 102:2365-2377. https://doi.org/10.3168/jds.2018-15466.

Pocrnic, I., D. A. Lourenco, Y. Masuda, A. Legarra, and I. Misztal. 2016. The dimensionality of genomic information and its effect on genomic prediction. Genetics 203:573-581. https://doi.org/10 .1534 /genetics.116.187013.

Quaas, R. L. 1988. Additive genetic model with groups and relationships. J. Dairy Sci. 71:1338-1345. https://doi.org/10.3168/jds .S0022-0302(88)79691-5.

Strandén, I., R. Evans, and E. A. Mäntysaari. 2019. Single-step evaluation for calving traits with 1.5 million genotypes: APY and ssGTBLUP approaches. Abstract from 70th Annual Meeting of the European Federation of Animal Science EAAP 2019, Ghent, Belgium.

Strandén, I., and D. J. Garrick. 2009. Derivation of equivalent computing algorithms for genomic predictions and reliabilities of animal merit. J. Dairy Sci. 92:2971-2975. https://doi.org/10.3168/ jds.2008-1929.
Strandén, I., and E. A. Mäntysaari. 2014. Comparison of some equivalent equations to solve single-step GBLUP. Page 22 in Proc. 10th World Congress on Genetics Applied to Livestock Production, Vancouver, Canada.

Strandén, I., K. Matilainen, G. P. Aamand, and E. A. Mäntysaari. 2017. Solving efficiently large single-step genomic best linear unbiased prediction models. J. Anim. Breed. Genet. 134:264-274. https://doi.org/10.1111/jbg.12257.

Taskinen, M., E. A. Mäntysaari, and I. Strandén. 2017. Single-step SNP-BLUP with on-the-fly imputed genotypes and residual polygenic effects. Genet. Sel. Evol. 49:36. https://doi.org/10.1186/ s12711-017-0310-9.

Tsuruta, S., D. A. L. Lourenco, Y. Masuda, I. Misztal, and T. J. Lawlor. 2019. Controlling bias in genomic breeding values for young genotyped bulls. J. Dairy Sci. 102:9956-9970. https://doi.org/10 .3168/jds.2019-16789.

Vandenplas, J., M. P. Calus, H. Eding, and C. Vuik. 2019. A secondlevel diagonal preconditioner for single-step SNPBLUP. Genet. Sel. Evol. 51:30. https://doi.org/10.1186/s12711-019-0472-8.

VanRaden, P. M. 2008. Efficient methods to compute genomic predictions. J. Dairy Sci. 91:4414-4423. https://doi.org/10.3168/jds .2007-0980.

VanRaden, P. M., M. E. Tooker, J. R. O'connell, J. B. Cole, and D. M. Bickhart. 2017. Selecting sequence variants to improve genomic predictions for dairy cattle. Genet. Sel. Evol. 49:32. https://doi .org/10.1186/s12711-017-0307-4.

Westell, R. A., R. L. Quaas, and L. D. Van Vleck. 1988. Genetic groups in an animal model. J. Dairy Sci. 71:1310-1318. https:// doi.org/10.3168/jds.S0022-0302(88)79688-5.

Winkelman, A. M., D. L. Johnson, and B. L. Harris. 2015. Application of genomic evaluation to dairy cattle in New Zealand. J. Dairy Sci. 98:659-675. https://doi.org/10.3168/jds.2014-8560.

\section{ORCIDS}

E. A. Mäntysaari ๑ https://orcid.org/0000-0003-0044-8473

M. Koivula (1) https://orcid.org/0000-0002-8793-7618

I. Strandén @ https://orcid.org/0000-0003-0161-2618 